\title{
Correction to: Phenotypic, Genetic, and Cytogenetic Evidence of Hybridization between Species of Trans-Andean Tamarins (Genus Saguinus)
}

\author{
Iván Darío Soto-Calderón ${ }^{1}$ (D) - Yuliet Andrea Acevedo-Garcés ${ }^{1}$. \\ Tatiana Restrepo-Agudelo ${ }^{2,3}$ • Julio César Llinás-Guerrero ${ }^{4}$. \\ Yúdrum Rivillas-Puello ${ }^{5}$. Juan Bautista López ${ }^{5}$
}

Published online: 7 February 2019

(C) Springer Science+Business Media, LLC, part of Springer Nature 2019

Correction to: Int J Primatol (2018) 39:1022-1038 https://doi.org/10.1007/s10764-018-0044-8

This article is intended for inclusion in the Special Issue on Primate Hybridization and Hybrid Zones. It was however published in a separate issue, Volume 39 Issue 6 and is available at https://doi.org/10.1007/s10764-018-0044-8.

The online version of the original article can be found at https://doi.org/10.1007/s10764-018-0044-8

Iván Darío Soto-Calderón

ivan.soto@udea.edu.co

1 Genética, Mejoramiento y Modelación Animal, Universidad de Antioquia, Calle 67 No. 53 - 108, Medellín, Antioquia, Colombia

2 Corporación Autónoma Regional del Valle del Cauca, Carrera 56 No. 1B -, 11 Cali, Valle del Cauca, Colombia

3 Present address: Área Metropolitana del Valle de Aburrá, Carrera 53 No. 40A - 31, Medellín, Antioquia, Colombia

4 Corporación Autónoma Regional de la Frontera Nororiental, Calle 13 No. 3E -, 278 Cúcuta, Norte de Santander, Colombia

5 Universidad Nacional de Colombia - Sede Medellín, Facultad de Ciencias, Calle 59A No. 63 - 20, Medellín, Colombia 